УДК 616.896+616.899.2-053.2|4|(571.51):159.938.363.3:159.924.7:316.344.233:347.639

Для цитирования: Максимова И.В., Горина Я.В., Дятловский В.В., Имамалиева А.Н., Лопатина О.Л., Пичугина Ю.А. Особенности социального положения пациентов детского возраста с расстройствами аутистического спектра, проживающих в Красноярске. Сибирский вестник психиатрии и наркологии. 2020; 1 (106): 49-55. https://doi.org/10.26617/1810-3111-2020-1(106)-49-55

\title{
Особенности социального положения пациентов детского возраста с расстройствами аутистического спектра, проживающих в Красноярске
}

\author{
Максимова И.В., Горина Я.В., Дятловский В.В., \\ Имамалиева А.Н., Лопатина О.Л., Пичугина Ю.А.
}

Красноярский государственный медицинский университет имени профессора В.Ф. Войно-Ясенецкого Россия, 660022, Красноярск, ул. Партизана Железняка, 1

\section{PEЗЮME}

Цель: изучить особенности социального положения пациентов детского возраста (от 4 до 11 лет) с аутистическими расстройствами, госпитализированных в психиатрический стационар впервые. Материал исследования: сформированы две группы пациентов. Основная группа состояла из 39 пациентов (31 мальчик и 8 девочек) с клиническими признаками расстройств аутистического спектра, их средний возраст составил 7,26 $\pm 1,75$ года. В группу сравнения вошли 74 человека (56 мальчиков и 18 девочек) с

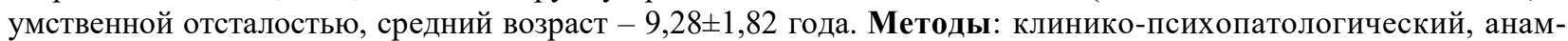
нестический, экспериментально-психологический. Результаты. Получены данные, что дети с аутизмом реже, чем пациенты с умственной отсталостью, относились к категории социальных сирот и находились на полном государственном обеспечении, реже проживали с опекунами или с родителями, злоупотребляющими психоактивными веществами. При этом коэффициент корреляции развития тяжелой патологии у пациентов с ранним детским аутизмом был выше, чем у больных умственной отсталостью с такими же характеристиками семейного окружения и материального положения. Заключение. Сделан вывод о превалировании детей, воспитывающихся в более благополучной социальной обстановке среди пациентов с аутизмом, чем среди пациентов с умственной отсталостью. Полученные результаты расширяют возможности психосоциальной реабилитации детей с расстройствами аутистического спектра за счет эффективного использования благоприятного семейного окружения.

Ключевые слова: расстройства аутистического спектра, умственная отсталость, детский возраст, социальный статус, состав семьи, материальное положение.

\section{ВВЕДЕНИЕ}

Современная оценка распространенности расстройств аутистического спектра указывает на увеличение числа пациентов с аутистическими расстройствами [1]. Как правило, показатели до 3-4 случаев на 10000 населения отражают распространенность типичных форм аутистических расстройств, таких как ранний детский аутизм и синдром Каннера [2]. При более широкой оценке иных нарушений, входящих в группу общих расстройств психического развития, показатели распространенности заметно повышаются - от 7,6 на 1000 до 157 на 10000 [3, 4].

В Красноярске в период с 2016 г. по 2018 г. число впервые зарегистрированных случаев аутистических расстройств выросло на 27,5\% с 323 до 412 случаев. Такая динамика отмечалась на фоне снижения как общего числа впервые выявленных психических расстройств - на 44,5\% (с 16048 до 8 908), так и на фоне сниже- ния в группе умственной отсталости - на 41,6\% (с 1853 до 1082 случаев) среди детского контингента населения за этот же период времени [5]. Значительный рост пациентов с аутизмом детского и более старших возрастов приводит к актуализации научных исследований, посвященных социальным проблемам этой категории больных [6]. Авторы публикуемых работ приходят к выводу о значительном ограничении их социальной интеграции [7], низком уровне занятости и условий жизни [8], наличии психологического дистресса, связанного с недостаточной социальной поддержкой [9]. Некоторые исследователи указывают на связь выраженности аутистических проявлений у взрослых с тяжестью первоначального диагноза в детском возрасте, с низким уровнем интеллекта, слабым социальным и языковым развитием, ограниченным доступом к услугам и программам социальной защиты [10]. 
Кроме того, в литературе встречаются данные, указывающие на наличие связи аутистических расстройств с социальным положением семей аутистов. Есть исследования, сообщающие о наименьшем числе случаев детского аутизма в тех семьях, где родители имеют низкий образовательный уровень [11], и демонстрирующие значительное преобладание пациентов с аутизмом, характеризующихся высоким социально-экономическим статусом, среди участников исследования в странах с высоким уровнем дохода [12]. В семьях этих пациентов отмечается высокая активность родителей в плане поиска и использования альтернативных вариантов развивающих программ, требующих от них собственных материальных вложений [13]. В одной из научных работ, выполненной китайскими исследователями, представлены данные, что дети в семьях с социальноэкономическим неблагополучием в виде недостаточного семейного дохода и низкого образования имеют больший риск развития детского аутизма [4].

Таким образом, противоречивость в публикуемых изданиях мнений о связи аутистических расстройств и социального положения пациентов сохраняет высокую актуальность исследований в этой области.

\section{ЦЕЛЬ ИССЛЕДОВАНИЯ}

Изучить социальные особенности пациентов с аутистическими расстройствами детского возраста, госпитализированных в психиатрический стационар впервые.

\section{МАТЕРИАЛЫ И МЕТОДЫ}

Для решения поставленных задач были сформированы две группы детей в возрасте от 4 до 11 лет, впервые проходивших обследование и лечение в стационарных условиях детского отделения Красноярского краевого психоневрологического диспансера № 1. Основную группу составили 39 пациентов (31 мальчик и 8 девочек) с клиническими признаками расстройств аутистического спектра,

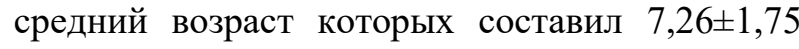
года. В группу сравнения вошли 74 ребенка (56 мальчиков и 18 девочек) с умственной отсталостью, их средний возраст составил $9,28 \pm 1,82$ года. Все дети имели показатель IQ ниже 70 баллов.

Клиническая картина психических расстройств, наличие или отсутствие аутистических особенностей коммуникации и поведения оценивались по критериям МКБ-10. На основе клинико-анамнестического метода уточнялись особенности семейного окружения и социаль- ного положения детей, характер материальнобытовых условий проживания, наличие родителей, злоупотребляющих психоактивными веществами. Степень выраженности аутистических черт определялась в соответствии с адаптированной версией шкалы CARS [14]. Для оценки интеллектуального развития детей использовался проективный личностный тест «Рисунок человека», так как у некоторых пациентов, включенных в исследовательскую выборку, невозможно было определить уровень интеллекта по стандартной методике в связи с аутистическими особенностями или вследствие выраженных поведенческих нарушений. Это позволило соотнести степень развития познавательных функций обследованных детей со 100-балльной системой IQ-теста.

Статистическая обработка данных проводилась с помощью программы Microsoft Office Excel 2013. Для оценки параметрических данных применялись методы описательной статистики, вычислялись средние значения параметров $\mathrm{M} \pm \mathrm{m}$. Уровень статистической значимости определялся с применением критерия $\chi^{2}$ Пирсона с поправкой Йейтса и точного критерия Фишера (двустороннего). Оценка связи между показателями проводилась с помощью коэффициента ранговой корреляции Спирмена.

Родителями или опекунами, являвшимися законными представителями пациентов, было подписано согласие на участие в исследовании на основе полной информации в соответствии с требованиями Закона РФ «О психиатрической помощи и гарантиях прав граждан при ее оказании». Отдельно были получены письменные согласия на обработку персональных данных. Организация и методы исследования были одобрены Этическим комитетом.

\section{РЕЗУЛЬТАТЫ И ОБСУЖДЕНИЕ}

Оценка особенностей социального статуса выявила определенные различия между пациентами основной группы (с расстройствами аутистического спектра) и группы сравнения (с умственной отсталостью). Показатели социального положения (наличие инвалидности, отсутствие родителей и опекунов, неполная семья в анамнезе, воспитание опекуном, употребление ПАВ родителями) пациентов обеих групп представлены в таблице 1 .

По результатам сравнительного анализа дети с аутизмом достоверно чаще имели группу инвалидности, чем дети с умственной отсталостью $(53,3 \%$ и $13,5 \%, \mathrm{p}<0,05)$, а также достоверно реже являлись социальными сиротами и 
находились на полном государственном обеспечении к моменту первой госпитализации $(2,6 \%$ и $35,1 \%$, p<0,05). Обнаружено, что относительно сопоставимое количество детей обеих групп проживали в неполных семьях (20,5\% и $25,7 \%)$; статистическая значимость соответствовала уровню случайных различий по этому показателю. Была отмечена явная тенденция к сохранению более благополучного положения детей-аутистов. Пациенты с расстройствами аутистического спектра реже, чем пациенты с умственной отсталостью, находились под опекой $(5,1 \%$ против $21,6 \%)$, а также реже имели родителей с алкогольной или наркотической зависимостью $(7,7 \%$ против $20,3 \%)$. Однако оценка статистической значимости с целью признания существенности различий между группами по этим параметрам не достигла статистически значимого уровня.

Т а б л и ц а 1 . Распределение показателей социального и семейного положения пациентов основной группы (с клиническими признаками расстройств аутистического спектра) и группы сравнения (с умственной отсталостью)

\begin{tabular}{|c|c|c|c|c|c|c|}
\hline \multirow[t]{2}{*}{ Показатель } & \multicolumn{2}{|c|}{ Группы } & \multirow{2}{*}{$\begin{array}{c}\text { Критерий } \chi^{2} \\
\text { Пирсона } \\
\text { с поправкой } \\
\text { Йейтса }\end{array}$} & \multirow{2}{*}{$\begin{array}{c}\text { Критиче- } \\
\text { ское значе- } \\
\text { ние } \chi^{2}\end{array}$} & \multirow{2}{*}{$\begin{array}{c}\text { Точный кри- } \\
\text { терий Фише- } \\
\text { ра (двусто- } \\
\text { ронний) }\end{array}$} & \multirow{2}{*}{$\begin{array}{c}\text { Уровень } \\
\text { значимости }\end{array}$} \\
\hline & $\begin{array}{l}\text { основная } \\
\text { абс. }(\%) \\
\text { n=39 }\end{array}$ & $\begin{array}{c}\text { сравнения } \\
\text { абс. }(\%) \\
\text { n=74 }\end{array}$ & & & & \\
\hline $\begin{array}{l}\text { Наличие группы инвалидности } \\
\text { к моменту госпитализации }\end{array}$ & $21(53,8 \%)$ & $10(13,5 \%)$ & 18,893 & 6,635 & 0,00001 & $\mathrm{p}<0,05$ \\
\hline $\begin{array}{l}\text { Отсутствие родителей и опекунов } \\
\text { (сироты на полном государствен- } \\
\text { ном обеспечении) }\end{array}$ & $1(2,6 \%)$ & $26(35,1 \%)$ & 13,162 & 6,635 & 0,00005 & $\mathrm{p}<0,05$ \\
\hline Воспитание в неполной семье & $8(20,5 \%)$ & $19(25,7 \%)$ & 0,144 & 0,705 & 0,64543 & $p>0,05$ \\
\hline Воспитание опекуном & $2(5,1 \%)$ & $16(21,6 \%)$ & 0,134 & 0,715 & 0,55476 & $p>0,05$ \\
\hline Родители употребляют ПАВ & $3(7,7 \%)$ & $15(20,3 \%)$ & 2,151 & 0,143 & 0,10699 & $p>0,05$ \\
\hline
\end{tabular}

На следующем этапе исследования для выявления показателей с достаточным уровнем статистической значимости были определены коэффициенты корреляции, позволяющие выделить ведущие факторы и связи, оказывающие максимальное влияние на получение детьми статуса инвалида детства.
Группа детей-сирот представляла собой малую выборку, недостаточную для проведения отдельного корреляционного анализа. Их данные учитывались при сравнении показателей пациентов - выходцев из неполных семей. Полученные результаты приведены в таблице 2 .

Т а б л и ц а 2. Коэффициенты корреляции наличия группы инвалидности с семейным положением для различных материально-бытовых условий положения пациентов с расстройствами аутистического спектра и умственной отсталостью

\begin{tabular}{|l|c|c|c|c|}
\hline \multicolumn{1}{|c|}{ Группа } & \multicolumn{2}{|c|}{ Полная семья } & \multicolumn{2}{|c|}{ Неполная семья } \\
\cline { 2 - 5 } & $\begin{array}{c}\text { хорошие матери- } \\
\text { ально-бытовые } \\
\text { условия }\end{array}$ & $\begin{array}{c}\text { плохие матери- } \\
\text { аль-бытовые } \\
\text { условия }\end{array}$ & $\begin{array}{c}\text { хорошие матери- } \\
\text { ально-бытовые } \\
\text { условия }\end{array}$ & $\begin{array}{c}\text { ально-бытовые } \\
\text { условия }\end{array}$ \\
\hline $\begin{array}{l}\text { Основная группа (пациенты с рас- } \\
\text { стройствами аутистического спектра) }\end{array}$ & 0,427 & 0,11 & $-0,55$ & $-0,166$ \\
\hline $\begin{array}{l}\text { Группа сравнения (пациенты } \\
\text { с умственной отсталостью) }\end{array}$ & $-0,078$ & $-0,044$ & $-0,076$ & $-0,088$ \\
\hline
\end{tabular}

Положительные корреляционные связи с наибольшим значением были установлены между полной семьей, хорошими материальнобытовыми условиями и наличием группы инвалидности у детей с аутизмом к моменту первой госпитализации. Для пациентов с умственной отсталостью с такими же показателями состава семьи и материально-бытовыми условиями коэффициенты корреляции с группой инвалидности имели отрицательные значения, указываю- щие, вероятно, на ухудшение семейного положения в связи с развитием тяжелой психической патологии у ребенка.

Неоднозначность оценки роли взаимоотношений в семье и социального положения пациентов приводит к актуализации поиска биологических механизмов, раскрывающих суть болезненного процесса, повышающих объективность изучения данной патологии. 
Одним из перспективных научных направлений, позволяющих вести исследования на современном уровне в данном русле, является определение влияния центральных эффектов окситоцина на социальное поведение, на формирование характерных признаков аутизма. Опубликованные результаты исследований роли окситоцина в регуляции социальной памяти и регуляции механизмов узнавания лиц [15], указание на снижение концентрации окситоцина в плазме крови при аутизме [16] - всё это открывает новые возможности для поиска нейробиологических механизмов развития данной патологии.

Заметные межгрупповые различия социальной активности и устойчивости внутрисемейных связей диктуют необходимость дальнейшего изучения пациентов выделенных групп в плане сопоставления показателей биологической регуляции поведения и выявленных социально-психологических особенностей.

\section{ЗАКЛЮЧЕНИЕ}

По результатам проведенного исследования было установлено, что пациенты детского возраста с расстройствами аутистического спектра, впервые госпитализированные в психиатрический стационар, достоверно чаще были выходцами из более благополучных семей, чем пациенты с умственной отсталостью. При этом риск развития психической патологии, требующей мер социальной поддержки и установления группы инвалидности, у пациентов с РДА оказался выше, чем у пациентов с умственной отсталостью с идентичными характеристиками семейного окружения и материального положения. Учитывая это, врачи-психиатры, педагоги и социальные работники могут рекомендовать для детей с аутизмом более сложные программы индивидуальной реабилитации или обучения, чем детям с умственной отсталостью такого же возраста и интеллекта. Определенные нами коэффициенты корреляции подтверждают тот факт, что родители пациентов с расстройствами аутистического спектра последовательнее и настойчивее выполняют рекомендации, направленные на максимальный результат в социализации и абилитации своих больных детей.

Представленные данные будут использованы в процессе реализации научного проекта по изучению роли окситоцина в регуляции социального поведения у пациентов с расстройствами аутистического спектра.
Результаты этой работы помогут с новой позиции, с опорой на конкретные показатели гормонального статуса, описать механизмы нарушений коммуникации и социального взаимодействия, обеспечат возможность для создания и внедрения в практику нового метода, значительно повышающего достоверность и прогностическую значимость результатов обследования детей с аутизмом.

\section{КОНФЛИКТ ИНТЕРЕСОВ}

Авторы заявляют об отсутствии явных и потенциальных конфликтов интересов в связи с публикацией данной статьи.

\section{ИСТОЧНИК ФИНАНСИРОВАНИЯ}

Работа выполнена по гранту для молодых ученых и обучающихся (приказ № 203-осн. от 26.03.2019 ФГБОУ ВО КрасГМУ им. проф. В.Ф. Войно-Ясенецкого).

\section{СООТВЕТСТВИЕ ПРИНЦИПАМ ЭТИКИ}

Работа соответствует этическим стандартам Хельсинской декларации ВМА и одобрена этическим комитетом ФГБОУ ВО «Красноярский государственный медицинский университет им. проф. В.Ф. Войно-Ясенецкого» Минздрава России (протокол ЛЭК № 86/2018 от 08.11.2018 г.).

\section{ЛИТЕРАТУРА}

1. Fombonne E. Epidemiology of pervasive developmental disorders. Pediatric Research. 2009; 65 (6): 591-598. DOI: 10.1203/PDR.0b013e31819e7203

2. Newschaffer C.J., Croen L.A., Daniels J., Giarelli E., Grether J.K., Levy S.E., Mandell D.S., Miller L.A., Pinto-Martin J., Reaven J., Reynolds A.M., Rice C. E., Schendel D., Windham G. C. The epidemiology of autism spectrum disorders. Annual Review of Public Health. 2007; 28: 235-258. DOI: 10.1146/annurev.publhealth.28.021406.144007

3. Baxter A.J., Brugha T.S., Erskine H.E., Scheurer R.W., Vos T., Scott J.G. The epidemiology and global burden of autism spectrum disorders. Psychol. Med. 2015; 45 (3): 601-613. DOI: 10.1017/S003329171400172X

4. He P., Guo C., Wang Z., Chen G., Li N., Zheng X. Socioeconomic status and childhood autism: A population-based study in China. Psychiatry Res. 2018 Jan; 259: 27-31. DOI: 10.1016/j.psychres.2017.08.046

5. Государственный доклад о состоянии здоровья населения и деятельности здравоохранения Красноярского края в 2018 году. Красноярск: Министерство здравоохранения Красноярского края, 2019: 152.

6. Ruggieri V., Gómez J.L.C., Martínez M.M., Arberas C. Aging and Autism: Understanding, Intervention, and Proposals to Improve Quality of Life. Curr Pharm Des. 2019 Dec; 25(41): 4454-4461. DOI : 10.2174/1381612825666191204165117

7. Howlin P., Moss P., Savage S., Rutter M. Social outcomes in mid- to later adulthood among individu- 
als diagnosed with autism and average nonverbal IQ as children. J Am Acad Child Adolesc Psychiatry. 2013; 52: 572-81. DOI:10.1016/j.jaac.2013.02.017

8. Henninger N.A., Taylor J.L. Outcomes in adults with autism spectrum disorders: a historical perspective. Autism. 2013 Jan; 17: 103-16. DOI:10.1177/1362361312441266

9. Seymour M., Giallo R., Wood C.E. Perceptions of social support: comparisons between fathers of children with autism spectrum disorder and fathers of children without developmental disabilities. J Intellect Disabil Res. 2019 Dec 5. DOI: 10.1111/jir.12704

10. Sevaslidou I., Chatzidimitriou C., Abatzoglou G. The long-term outcomes of a cohort of adolescents and adults from Greece with autism spectrum disorder. Ann Gen Psychiatry. 2019 Nov 16; 18: 26. DOI: 10.1186/s12991-019-0250-6

11. Baird G., Simonoff E., Pickles A., Chandler S., Loucas T., Meldrum D., Charman T. Prevalence of disorders of the autism spectrum in a population cohort of children in South Thames: the Special Needs and Autism Project (SNAP). Lancet. 2006 Jul 15; 368 (9531): 210-215. DOI: 10.1016/S01406736(06)69041-7

12. Durkin M. S., Elsabbagh M., Barbaro J., Gladstone M., Happe F., Hoekstra R. A., Lee L. C., Rattazzi A., Stapel-Wax J., Stone W. L., Tager-Flusberg H., Thurm A., Tomlinson M., Shih A. Autism screening and diagnosis in low resource settings: Challenges and opportunities to enhance research and services worldwide. Autism Res. 2015 Oct; 8 (5): 473-6. DOI: 10.1002/aur.1575

13. Пичугина Ю.А., Сумароков А.А., Салмина А.Б., Хигашида X. Некоторые аспекты оказания помощи детям с аутизмом в условиях Красноярска. Сибирское медиџинское обозрение. 2009; 59 (5): 19-22.

14. Касаткин В.Н., Шапошникова А.Ф., Письменная Н.В., Бородина Л.Г., Сударикова М.А. Опыт применения стандартизированных диагностических оценочных шкал в комплексной программе лечебно-коррекционной помощи детям с расстройствами аутистического спектра. Детский аутизм: исследования и практика. М. : Региональная общественная организация «Образование и здоровье», 2008; 1: 26-58.

15. Kanat M., Spenthof I., Riedel A., van Elst L.T., Heinrichs M., Domes G. Restoring effects of oxytocin on the attentional preference for faces in autism. Transl Psychiatry. 2017 Apr 18; 7 (4): e1097. DOI: $10.1038 /$ tp.2017.67

16. Zhang H.F., Dai Y.C., Wu J., Jia M.X., Zhang J.S., Shou X.J., Han S.P., Zhang R., Han J.S. Plasma Oxytocin and Arginine-Vasopressin Levels in Children with Autism Spectrum Disorder in China: Associations with Symptoms. Neurosci Bull. 2016 Oct; 32(5): 423-32. DOI: 10.1007/s12264-0160046-5

Поступила в редакцию 21.10.2019 Утверждена к печати 27.01.2020

Максимова Ирина Викторовна - ассистент кафедры психиатрии и наркологии с курсом ПО КрасГMУ. ORCID iD 0000-0001-5076-5368. kafedra_pn@mail.ru

Горина Яна Валерьевна - кандидат фармацевтических наук, доцент кафедры биологической химии с курсом медицинской, фармацевтической и токсикологической химии КрасГМУ, научный сотрудник НИИ молекулярной медицины и патобиохимии КрасГМУ. ORCID iD 0000-0002-3341-1557. yana_20@bk.ru

Дятловский Вадим Владимирович - студент лечебного факультета КрасГМУ. kafedra_pn@mail.ru

Имамалиева Анжелика Нарынбаевна - студентка лечебного факультета КрасГМУ. kafedra_pn@mail.ru

Лопатина Ольга Леонидовна - кандидат биологических наук, доцент кафедры биологической химии с курсом медицинской, фармацевтической и токсикологической химии КрасГМУ, научный сотрудник НИИ молекулярной медицины и патобиохимии КрасГМУ, ORCID iD0000-0002-7884-2721; e-mail: ol.lopatina@gmail.com

Пичугина Юлия Анатольевна - кандидат медицинских наук, доцент кафедры психиатрии и наркологии с курсом ПО КрасГМУ. ORCID iD 0000-0001-8391-821X.

Пичугина Юлия Анатольевна, yulia651@mail.ru 
For citation: Maksimova I.V., Gorina Ya.V., Dyatlovskiy V.V., Imamalieva A.N., Lopatina O.L., Pichugina Yu.A. Features of the social status of children with autism spectrum disorders living in Krasnoyarsk. Siberian Herald of Psychiatry and Addiction Psychiatry. 2020; 1 (106): 49-55. https://doi.org/10.26617/1810-3111-2020-1(106)-49-55

\title{
Features of the social status of children with autism spectrum disorders living in Krasnoyarsk
}

\section{Maksimova I.V., Gorina Ya.V., Dyatlovskiy V.V., Imamalieva A.N., Lopatina O.L., Pichugina Yu.A.}

Krasnoyarsk State Medical University of a Name of the Professor V.F. Vojno-Jasenetsky Street Partizan Zheleznyak, 1, 660022, Krasnoyarsk, Russian Federation

\begin{abstract}
Objective: to study the social conditions of children of childhood (from 4 to 11 years old) with autistic disorders who were hospitalized in a psychiatric hospital for the first time. Study material: two groups of patients were formed. The main group consisted of 39 patients (31 boys and 8 girls) with clinical signs of autism spectrum disorders, their average age was $7.26 \pm 1.75$ years. The comparison group included 74 people (56 boys and 18 girls) with mental retardation, the average age was $9.28 \pm 1.82$ years. Methods: clinical, psychopathological, anamnestic, experimental psychological. Results. The data were obtained that children with autism were less likely than patients with mental retardation to belong to the category of social orphans and were on full state support, less likely to live with guardians or with parents who abuse psychoactive substances. Moreover, the correlation coefficient for the development of severe pathology in patients with early childhood autism was higher than in patients with mental retardation with the same characteristics of the family environment and financial situation. Conclusion. The conclusion is made that the prevalence of children brought up in a more favorable social environment among patients with autism than among patients with mental retardation. The obtained results expand the possibilities of psychosocial rehabilitation of children with autism spectrum disorders due to the effective use of a favorable family environment.
\end{abstract}

Keywords: children-orphans, children without parental care, psychopathological disorders, behavioral disorders, mental disorders, Trans-Baikal Territory.

\section{REFERENCES}

1. Fombonne E. Epidemiology of pervasive developmental disorders. Pediatric Research. 2009; 65 (6): 591-598. DOI: 10.1203/PDR.0b013e31819e7203

2. Newschaffer C.J., Croen L.A., Daniels J., Giarelli E., Grether J.K., Levy S.E., Mandell D.S., Miller L.A., Pinto-Martin J., Reaven J., Reynolds A.M., Rice C. E., Schendel D., Windham G. C. The epidemiology of autism spectrum disorders. Annual Review of Public Health. 2007; 28: 235-258. DOI: 10.1146/annurev.publhealth.28.021406.144007

3. Baxter A.J., Brugha T.S., Erskine H.E., Scheurer R.W., Vos T., Scott J.G. The epidemiology and global burden of autism spectrum disorders. Psychol. Med. 2015; 45 (3): 601-613. DOI: 10.1017/S003329171400172X

4. He P., Guo C., Wang Z., Chen G., Li N., Zheng X. Socioeconomic status and childhood autism: A population-based study in China. Psychiatry Res. 2018 Jan; 259: 27-31. DOI: 10.1016/j.psychres.2017.08.046

5. Gosudarstvennyy doklad o sostoyanii zdorov'ya naseleniya i deyatel'nosti zdravookhraneniya Krasnoyarskogo kraya v 2018 godu [State report on the state of public health and health care in the Krasnoyarsk Territory in 2018]. Krasnoyarsk: Ministry of
Health of the Krasnoyarsk Territory, 2019: 152 (in Russian).

6. Ruggieri V., Gómez J.L.C., Martínez M.M., Arberas C. Aging and Autism: Understanding, Intervention, and Proposals to Improve Quality of Life. Curr Pharm Des. 2019 Dec; 25(41): 4454-4461. DOI : $10.2174 / 1381612825666191204165117$

7. Howlin P., Moss P., Savage S., Rutter M. Social outcomes in mid- to later adulthood among individuals diagnosed with autism and average nonverbal IQ as children. J Am Acad Child Adolesc Psychiatry. 2013; 52: 572-81. DOI:10.1016/j.jaac.2013.02.017

8. Henninger N.A., Taylor J.L. Outcomes in adults with autism spectrum disorders: a historical perspective. Autism. 2013 Jan; 17: 103-16. DOI:10.1177/1362361312441266

9. Seymour M., Giallo R., Wood C.E. Perceptions of social support: comparisons between fathers of children with autism spectrum disorder and fathers of children without developmental disabilities. J Intellect Disabil Res. 2019 Dec 5. DOI: 10.1111/jir.12704

10. Sevaslidou I., Chatzidimitriou C., Abatzoglou G. The long-term outcomes of a cohort of adolescents and adults from Greece with autism spectrum disorder. Ann Gen Psychiatry. 2019 Nov 16; 18: 26. DOI: $10.1186 / \mathrm{s} 12991-019-0250-6$ 
11. Baird G., Simonoff E., Pickles A., Chandler S., Loucas T., Meldrum D., Charman T. Prevalence of disorders of the autism spectrum in a population cohort of children in South Thames: the Special Needs and Autism Project (SNAP). Lancet. 2006 Jul 15; 368 (9531): 210-215. DOI: 10.1016/S01406736(06)69041-7

12. Durkin M. S., Elsabbagh M., Barbaro J., Gladstone M., Happe F., Hoekstra R. A., Lee L. C., Rattazzi A., Stapel-Wax J., Stone W. L., Tager-Flusberg H., Thurm A., Tomlinson M., Shih A. Autism screening and diagnosis in low resource settings: Challenges and opportunities to enhance research and services worldwide. Autism Res. 2015 Oct; 8 (5): 473-6. DOI: 10.1002/aur.1575

13. Pichugina Yu.A., Sumarokov A.A., Salmina A.B., Higashida Kh. Nekotoryye aspekty okazaniya pomoshchi detyam s autizmom v usloviyakh Krasnoyarska [Some aspects of assisting children with autism in the conditions of Krasnoyarsk]. Sibirskoye meditsinskoye obozreniye - Siberian Medical Review. 2009; 59 (5): 19-22 (in Russian).

14. Kasatkin V.N., Shaposhnikova A.F., Pismennaya N.V., Borodina L.G., Sudarikova M.A. Opyt primeneniya standartizirovannykh diagnosticheskikh otsenochnykh shkal v kompleksnoy pro- gramme lechebno-korrektsionnoy pomoshchi detyam s rasstroystvami autisticheskogo spectra [The experience of using standardized diagnostic assessment scales in a comprehensive program of treatment and correctional care for children with autism spectrum disorders]. Childhood autism: research and practice. Moscow : Regional Public Organization "Education and Health", 2008; 1: 26-58 (in Russian).

15. Kanat M., Spenthof I., Riedel A., van Elst L.T., Heinrichs M., Domes G. Restoring effects of oxytocin on the attentional preference for faces in autism. Transl Psychiatry. 2017 Apr 18; 7 (4): e1097. DOI: $10.1038 /$ tp.2017.67

16. Zhang H.F., Dai Y.C., Wu J., Jia M.X., Zhang J.S., Shou X.J., Han S.P., Zhang R., Han J.S. Plasma Oxytocin and Arginine-Vasopressin Levels in Children with Autism Spectrum Disorder in China: Associations with Symptoms. Neurosci Bull. 2016 Oct; 32(5): 423-32. DOI: 10.1007/s12264-0160046-5

Received October 21.2019

Accepted January 27.2020

Maksimova Irina V. - Assistant of the Department of Psychiatry and Narcology with a vocational education course. Krasnoyarsk State Medical University of a Name of the Professor V.F. Vojno-Jasenetsky. ORCID iD 0000-00015076-5368. kafedra_pn@mail.ru

Gorina Yana V. - Candidate of Pharmaceutical Sciences, Associate Professor of the Department of Biological Chemistry with a course in medical, pharmaceutical and toxicological chemistry, Krasnoyarsk State Medical University of a Name of the Professor V.F. Vojno-Jasenetsky, Researcher, Research Institute of Molecular Medicine and Pathobiochemistry, Krasnoyarsk State Medical University of a Name of the Professor V.F. Vojno-Jasenetsky. ORCID iD 0000-0002-3341-1557. yana_20@bk.ru

Dyatlovskiy Vadim V. - student Faculty of Medicine, Krasnoyarsk State Medical University of a Name of the Professor V.F. Vojno-Jasenetsky. kafedra_pn@mail.ru

Imamalieva Anzhelika N. - student Faculty of Medicine, Krasnoyarsk State Medical University of a Name of the Professor V.F. Vojno-Jasenetsky. kafedra_pn@mail.ru

Lopatina Olga L. - Candidate of Biological Sciences, Associate Professor of the Department of Biological Chemistry with a course in medical, pharmaceutical and toxicological chemistry, Krasnoyarsk State Medical University of a Name of the Professor V.F. Vojno-Jasenetsky, Researcher, Research Institute of Molecular Medicine and Pathobiochemistry, Krasnoyarsk State Medical University of a Name of the Professor V.F. Vojno-Jasenetsky. ORCID iD00000002-7884-2721; e-mail: ol.lopatina@gmail.com

Pichugina Yuliya A. - Candidate of Medical Sciences, Associate Professor of the Department of Psychiatry and Narcology with a vocational education course, Krasnoyarsk State Medical University of a Name of the Professor V.F. Vojno-Jasenetsky. ORCID iD 0000-0001-8391-821X.

Pichugina Yuliya A., yulia651@ mail.ru 